\title{
Le double fardeau épidémiologique chez les personnes âgées en milieu rural africain: tendances de mortalité et causes de décès à Nouna (Burkina Faso)
}

\author{
Niamba Louis ${ }^{1 a, b}$, LeGrand Thomas ${ }^{a}$, Gagnon Alaina, Sié Alib, Zabré Pascal ${ }^{b}$ \\ a Département de Démographie, Université de Montréal, Canada \\ ${ }^{\text {b } C e n t r e ~ d e ~ R e c h e r c h e ~ e n ~ S a n t e ́ ~ d e ~ N o u n a, ~ B u r k i n a ~ F a s o ~}$
}

\begin{abstract}
This study aims to quantify the phenomenon of the double epidemiological burden in Burkina-Faso. Data from Nouna Health and Demographic Surveillance System (HDSS) were used with a total of 4427 deaths among those aged 50 and over, between 1993 and 2012 (including 2323 for which a cause is clearly diagnosed). The share of deaths due to communicable diseases did not significantly decline over time $(-13 \%$; $p$-value $=0.158)$ while the proportion of deaths from non-communicable causes increased significantly $(+178 \%$; $p$-value $<0.001)$. This resulted primarily from a rise in mortality rates from cardiovascular disease, especially among men. The rise of cardiovascular diseases led to a reduction in the life expectancy at age 50 (-2.65 years) between 1997-2004 and 2005-2012. Mortality from cardiovascular diseases contributes to the double epidemiological burden among the elderly in Burkina Faso.
\end{abstract}

Keywords: Communicable diseases; Non-communicable diseases; double epidemiological burden; Nouna; Burkina Faso

\section{Résumé}

Cette étude vise à quantifier le phénomène du double fardeau épidémiologique chez les personnes de plus de 50 ans au Burkina Faso. L'analyse porte sur les données de l'observatoire de population de Nouna, et en particulier sur un total de 4427 décès de personnes de 50 ans et plus survenus entre 1993 et 2012 . Pour environ la moitié de ces décès ( 2323 cas), une cause a pu être clairement diagnostiquée. L'analyse révèle que la part des décès due aux maladies transmissibles n'a pas significativement diminué $(-13 \%$; $p$-value $=0,158)$ alors que celle due aux maladies non transmissibles a significativement augmenté $(+178 \%$; $p$-value $<0,00 I)$. Ceci s'explique surtout par une hausse des taux de mortalité dus aux maladies cardiovasculaires, particulièrement chez les hommes. La montée des maladies cardiovasculaires a contribué à une baisse de l'espérance de vie à 50 ans, d'environ 2,6 ans entre 19972004 et 2005-2012. Cette forte mortalité due aux maladies cardiovasculaires contribue au double fardeau épidémiologique chez les personnes âgées au Burkina Faso.

Mots-clés: Maladies transmissibles; Maladies non transmissibles; double fardeau épidémiologique; Nouna; Burkina Faso

\footnotetext{
${ }^{1}$ Auteur des correspondances :louis.niamba@umontreal.ca, Département de Démographie, Université de Montréal, 3150 rue JeanBrillant, local C-5043, H3T1N8, Montréal (Québec), Canada.
} 


\section{Introduction}

Le niveau de mortalité par cause est un élément crucial dans la planification des interventions en matière de santé des populations (Murray et Lopez, 2012). Malheureusement, la connaissance des causes de décès reste très lacunaire en Afrique subsaharienne, du fait de la faible complétude de l'état civil (Garenne et al., 1999; Duthé et Pison, 2008; Soura et al., 20/4). Dans le contexte africain, la priorité de santé publique reste souvent largement centrée sur la santé de la mère et de l'enfant (Duthé et al., 2010).

Pourtant, les niveaux de mortalité aux âges adultes, et particulièrement au-delà de 50 ans, deviennent des indicateurs de santé incontournables, car on assiste de nos jours à une augmentation du nombre de personnes âgées en Afrique subsaharienne. Même si la proportion de personnes âgées de plus de 50 ans devrait rester relativement faible dans cette partie de l'Afrique ( $10 \%$ en 2015 et $15 \%$ en 2050 d'après les projections des Nations Unies (2014) sous l'hypothèse d'une moyenne fécondité), leur nombre absolu va plus que tripler d'ici à 2050 (94 292000 personnes en 2015 et 308 508000 personnes en 2050, toujours d'après ces projections). Le Burkina Faso est à cet égard assez représentatif du sous-continent car les personnes de 50 ans et plus constituent $8,3 \%$ de la population générale en 2015 et ce pourcentage atteindra 13,9\% en 2050. Par contre, le nombre de personnes âgées de plus de 50 ans va presque quadrupler dans ce pays d'ici à 2050, pour passer de I,5 millions en 2015 à 5,7 millions en 2050 (ONU, 20I4).

Ce nombre croissant de personnes âgées résulte de la transition démographique en cours, qui a entraîné une forte croissance des effectifs de population en raison d'un décalage entre la baisse de la mortalité et la baisse, plus timide, de la fécondité (Vallin, 2002). En Afrique, comme ailleurs dans le monde, l'allongement de l'espérance de vie contribue également à l'augmentation du nombre d'adultes et de personnes âgées. Un des corollaires de cette hausse de l'espérance de vie est l'augmentation du poids des maladies non transmissibles dans la population, qui résulte de la substitution des maladies infectieuses et parasitaires par des pathologies dites de dégénérescence et de société (cancers, maladies cardiovasculaires, troubles mentaux) (Omran, 1971; Aboderin, 20l0; Streatfield et al., 20l4b; Olshansky et Ault, 1986). Ce changement dans le profil des causes de décès a été qualifié par Omran de transition épidémiologique (Omran, 197I). Les déterminants de cette transition épidémiologique sont intimement associés à ceux de la transition démographique (baisse de la fécondité et de la mortalité) et sont généralement attribués à la «modernisation» (Soura et al., 20l4).

Étant donné que certaines maladies infectieuses continuent de causer de nombreux décès en Afrique (et même chez les personnes âgées), la transition épidémiologique y est actuellement caractérisée par un double fardeau de maladies (Tollman S.M. et al., 2008; Kuate Defo, 20I4; Maher et Sekajugo, 20II; Masquelier et al., 2014): les populations sont confrontées en même temps aux maladies transmissibles et celles non transmissibles. $\mathrm{Si}$ à l'échelle de la population générale ce double fardeau est attendu parce qu'il combine des schémas épidémiologiques différents selon les groupes d'âges, la situation parmi les adultes est difficile à cerner, et cette difficulté s'accroit considérablement pour les personnes âgées.

Dans ce contexte et disposant de données sur la mortalité et les causes de décès d'une population vivant en milieu rural ouest africain, nous avons ici pour objectif de mettre en évidence les changements épidémiologiques qui se sont opérés sur une période de 20 ans chez les personnes âgées de plus 50 ans. Pour ce faire, nous nous baserons simplement sur les niveaux de mortalité générale, les proportions et les taux de mortalité par cause parmi les plus de 50 ans au cours de la période étudiée (1993-20|2). Les maladies cardiovasculaires, respiratoires et les tumeurs retiendront notre attention car ces dernières sont considérées comme les maladies qui connaitront la plus forte augmentation au cours des prochaines années en Afrique (Aboderin, 20ll). Le paludisme étant la première cause de mortalité dans la zone (Ramroth et al., 20I2), nous y porterons également une attention particulière.

\section{Revue de la Littérature}

L'étude des changements dans la hiérarchie des causes de décès nous ramène aux travaux d'Omran (I97I) et S. Jay Olshansky et al (1986) sur la transition épidémiologique. Selon cette théorie, lorsque les nations se modernisent, elles ont tendance à améliorer leurs conditions sociales, économiques et sanitaires. Les conditions de vie qui étaient auparavant favorables à la propagation des maladies infectieuses et parasitaires sont rapidement remplacées par des meilleures conditions sanitaires. Le risque de mourir de maladies infectieuses étant réduite pour la population, de nombreuses personnes survivent jusqu'à des âges avancés et meurent de maladies de dégénérescence ou de société (cancer, maladies cardiovasculaires, diabète, démences séniles, suicides, accidents, etc...) (Olshanky et Ault, 1986, Omran, 197I). 
Omran (197I) a résumé cette transition épidémiologique en trois étapes essentielles. La première est «l'âge de la pestilence et de la famine»; les niveaux de mortalité y étaient élevés, l'espérance de vie oscillait entre 20 et 40 ans et les principales causes de décès comprenaient la grippe, la pneumonie, la diarrhée, la variole, la tuberculose et d'autres maladies connexes. La deuxième étape est celle de l'«âge du recul des pandémies»; c'est véritablement l'étape de transition lors de laquelle l'espérance de vie atteint environ 50 ans. La dernière étape, «l'âge des maladies de dégénérescence et des maladies de société» est caractérisée par un ralentissement des progrès de l'espérance de vie, de par la survenue de nouvelles maladies que sont les maladies de dégénérescence ou les maladies de société.

Cependant, très vite, ce schéma d'Omran a été contrarié par l'épreuve des faits. Dans tous les pays occidentaux, l'espérance de vie est repartie à la hausse dès le début des années 1970, sous l'effet d'un recul massif des maladies cardio-vasculaires (Vallin et Meslé, 2010). C'est alors que Jay Olshansky et Brian Ault (1986) puis Richard Rogers et Robert Hackenberg (1987) ont évoqué une "quatrième phase » de la transition épidémiologique, la révolution cardio-vasculaire (Olshanky et Ault, 1986; Rogers et Hackemberg, 1987).

L'application aux pays africains de cette transition épidémiologique décrite par Omran (197I) fait également l'objet de débats, même si les analyses en la matière se sont souvent confrontées à la rareté des données et au manque des sources écrites en matière d'histoires démographiques et épidémiologiques (Maher et Sekajugo, 20II; Mackenbach, 1994; Tabutin et Schoumaker, 2004). Vallin et Meslé (2010) observent notamment que cette théorie de la transition épidémiologique s'est heurtée à des obstacles durables en Afrique sub-saharienne. Ils évoquent trois handicaps essentiels: I) l'élan brisé par la crise économique mondiale des années 1970 et les plans d'ajustement structurels des années 1980;2) l'insuffisance des progrès sanitaires dans ces pays et 3), l'épidémie du $\mathrm{VIH} /$ sida qui a sévèrement touché beaucoup de pays subsahariens. Ainsi, l'espérance de vie a pu reculer pour un temps, pour ensuite enregistrer des progrès dans plusieurs pays, grâce aux succès dans la lutte contre cette pandémie (Salomon et al., 20I2). Les changements dans la hiérarchie des causes de décès restent également modestes. En Afrique subsaharienne, les maladies transmissibles causaient environ 2,5 fois plus de décès que les maladies chroniques en 1990 et presque 20 ans après, soit en 2008, ce ratio est demeuré le même (Kuate Defo, 20I4). Kuate Defo (20I4) ajoute à ces trois principaux obstacles les conflits et les instabilités sur le continent africain qui ont ralenti gravement et durablement depuis l'ère postcoloniale les progrès sociaux, économiques, politiques et sanitaires. II conclut que l'Afrique sub-saharienne n'est pas, sauf quelques exceptions près, embarquée dans la transition épidémiologique décrite par Omran (I97I).

Certains auteurs, comme Garenne et al. (1999) et De-Graft Aikins et al (20l0), adoptent une position moins tranchée. Selon eux, il n'y a pas de doute que les pays africains suivent également le chemin indiqué par Omran (I97I) même s'il faut relever quelques exceptions importantes. En effet, les niveaux de mortalité et les causes de décès évoluent très rapidement en Afrique, conséquence des nombreuses actions menées pour améliorer la santé et la croissance économique. La spécificité de l'Afrique sub-saharienne tient surtout au fait qu'à l'heure actuelle, l'Afrique doit affronter en même temps les maladies de la pauvreté et celles du développement (Garenne et al., 1999; De-Graft Aikins et al., 20l0). Si les maladies infectieuses restent une préoccupation importante en santé publique, tout indique que la mortalité associée aux maladies chroniques telles que les maladies cardiovasculaires et les tumeurs compte pour une proportion croissante des décès. En effet, si les maladies chroniques constituent depuis longtemps la première cause de mortalité dans les pays industrialisés ( $88 \%$ des décès), on peut déjà leur attribuer environ $25 \%$ des décès en Afrique subsaharienne en 2010 . Cette proportion s'élève à $54 \%$ chez les personnes de 50 à 69 ans $^{2}$.

$\mathrm{Si}$ au niveau de l'Afrique de façon générale, il existe des estimations (qui s'appuient très souvent sur des modélisations statistiques) permettant de documenter les tendances en matière de transition épidémiologique, les études locales et nationales sur les causes de décès sont rares (Soura et al., 2014, Kuate Defo, 2014, Sie et al., 20l0). Dans certaines régions où des données existent, et notamment en milieu urbain comme à Addis Abéba en Éthiopie, les décès attribués aux maladies chroniques sont majoritaires après 15 ans (5l\%) (Awoke et Damen, 2012). En milieu rural du KwaZulu-Natal en Afrique du sud, Herbst et ses collègues (20l I) estiment que $56 \%$ des décès sont imputables à des maladies transmissibles (dont $50 \%$ causés par le $\mathrm{VIH} /$ sida), $21 \%$ aux maladies non transmissibles, le solde étant dû à des blessures ou des causes indéterminées.

Si la plupart des recherches sont unanimes sur le fait qu'il y a bien un changement dans la hiérarchie des causes de décès au fil des années en Afrique, les

2 Sc: GBD 2010 - http://vizhub.healthdata.org/gbdcompare/ 
connaissances sur la nature et l'intensité de ces changements restent rudimentaires. Dans beaucoup de pays, le manque de données sur les causes spécifiques de mortalité constitue un handicap pour le suivi des indicateurs dans ce domaine. Dans ce contexte, les systèmes de surveillance démographique et de santé (ou observatoires), qui collectent de façon régulière des données de morbidité et de mortalité sur la base des déclarations des individus interrogés viennent partiellement combler ce déficit (Sie et al., 2010; Duthé et Pison, 2008).

Au Burkina Faso, plusieurs travaux ont fait usage de ces données d'observatoires pour caractériser la transition épidémiologique en cours. On citera notamment les recherches récentes de Soura et al (20l4) et de Rossier et al (20/4), qui comparent les causes de décès entre les quartiers lotis et non lotis de Ouagadougou. Ils montrent que pour tous âges confondus, les maladies transmissibles restent la première catégorie de causes de décès dans l'observatoire de Ouagadougou. Les maladies transmissibles demeurent la principale cause de décès chez les moins de 15 ans dans les deux types de quartiers, tandis que les maladies non transmissibles constituent les principales causes de mortalité des plus de 50 ans, et leur part est plus élevée dans les quartiers lotis (Soura et al., 20l4; Rossier et al., 20/4). Streatfield et al. (20/4) fournissent également un aperçu général des tendances des taux de mortalité dus aux maladies non transmissibles chez les adultes de 15 ans et plus dans les observatoires membres du réseau INDEPTH. Pour les observatoires de Ouagadougou et de Nouna, ils ont constaté que les tumeurs et les maladies cardiovasculaires faisaient partie des cinq maladies non transmissibles les plus fréquentes chez les adultes de 15 ans et plus.

Notre étude vient compléter ces travaux, grâce aux données de l'observatoire de Nouna. Elle vise à contribuer à une meilleure connaissance de la répartition et de l'intensité des maladies respiratoires, cardiovasculaires, des tumeurs et du paludisme chez les personnes de plus de 50 ans en milieu rural africain. Au-delà des proportions qui ont été calculées dans les études précédentes, ce travail examine la variation dans les taux de mortalité due à ces maladies spécifiques. Nous mettons en évidence l'effet de ces changements d'intensité sur l'espérance de vie de ces personnes âgées de plus de 50 ans.

\section{Données et Méthodes Données}

La source de données qui est utilisée dans cette étude est celle de l'observatoire géré par le Centre de Recherche en Santé de Nouna (CRSN) dans le district de Santé du même nom au Burkina Faso. La zone de Nouna est située à environ $300 \mathrm{~km}$ au sudouest de Ouagadougou. Cette zone est sous surveillance démographique et sanitaire depuis une vingtaine d'années. La population couverte au 31 / 2 /201 2 était de 93667 habitants. A cette même date, la population était à majorité rurale (7/\%), avec une densité de 55,8 habitants $/ \mathrm{km}^{2}$. Parmi les informations régulièrement collectées depuis le début du suivi, se trouvent les décès et leurs causes probables déterminées par la méthode des autopsies verbales. La population cible dans cette étude est constituée de toutes les personnes âgées de 50 ans et plus ayant résidé à un moment donné dans la zone d'étude entre 1993 et 2012 .

\section{Procédure des autopsies verbales}

Dans les pays moins développés, de nombreux décès ont lieu à domicile et sans assistance médicale (Soura et al., 20l4). L'information sur les causes de décès dans ces pays est donc fragmentaire (Duthé et Pison, 2008; Streatfield et al., 2014a). Au Burkina Faso, on estime que seul I décès sur 13 est enregistré par une formation sanitaire (Baya, 2004). Dans ce contexte, les observatoires utilisent des autopsies verbales pour collecter des informations sur les causes de décès. La méthode est basée sur des questionnaires permettant de retracer l'histoire de la maladie, sa durée, les symptômes et les traitements. A partir de la séquence et de la combinaison des symptômes et des faits déclarés, deux médecins déterminent généralement de manière non concertée, la cause probable du décès; en cas de divergence entre ces deux pronostics, un troisième médecin est invité à se prononcer. Dans le cas d'une troisième opinion divergente, la cause de décès est alors classée comme inconnue. La cause probable du décès retenue et notée est celle qui est donnée par au moins deux médecins. La $10^{e}$ révision de la classification internationale des maladies (CIMIO) (OMS, 1993-1996) a été utilisée pour codifier les causes de décès dans cette étude.

Dans l'observatoire de Nouna, sur la période 1993-2012, 4427 décès survenus au-delà de 50 ans ont été recensés. Pour 294I d'entre eux $(66,4 \%)$, des autopsies verbales ont pu être réalisées. Les décès pour lesquels les autopsies n'ont pu être faites sont surtout des cas d'émigration ou d'absence des proches lors de l'entretien. Dans le cas de décès d'adultes, la dissolution du ménage est fréquente et elle complique la conduite d'autopsies verbales auprès des anciens membres de ce ménage. Sur les $294 \mathrm{I}$ autopsies verbales, 2323 (79\%) ont abouti à un diagnostic clair, et 621 décès $(21 \%)$ ont été classés parmi les décès de cause indéterminée ou inconnue. 


\section{Analyse statistique}

Dans un premier temps, les taux de mortalité par groupe d'âges et pour chaque sexe sont calculés pour l'ensemble de la période. Les tendances de l'espérance de vie à 50 ans sont estimées pour cinq périodes de quatre ans: 1993-1996, 1997-2000, 200 I-2004 ; 2005-2008 ; 2009-20I 2.

Dans un second temps, nous mettons en évidence l'évolution des proportions des grands groupes de causes de décès (maladies transmissibles; non transmissibles ; accidents et mortalité violente).

Ensuite, nous mettons l'accent sur quatre catégories de causes; les maladies cardiovasculaires, les maladies respiratoires, les tumeurs et le paludisme. Les taux de mortalité par ces causes spécifiques sont ajustés pour tenir compte de la présence de décès dont la cause est manquante. Cet ajustement suppose que la répartition des décès par cause est la même parmi les décès dont la cause est connue et parmi ceux dont la cause est inconnue (Rowe, 2006). Afin de comparer les taux selon les différentes périodes, nous utiliserons le pourcentage de variation relative (VR) entre les taux ajustés de décès. Par exemple :

$$
V R_{2009-2012}^{1993-1996}=\frac{\operatorname{Taux}(2009-2012)-\operatorname{Taux}(1993-1996)}{\operatorname{Taux}(1993-1996)} *
$$
100.

Étant donné que les taux de mortalité des différentes périodes peuvent être influencés par les changements dans la structure par âge, les taux ont tous été ajustés pour l'âge. Pour ce faire, nous avons utilisé comme structure standard celle de notre population en 1993.

Enfin, nous avons évalué la contribution des causes spécifiques de décès aux variations de l'espérance de vie à 50 ans. Pour estimer ces contributions selon les périodes, nous faisons appel à la méthode développée par Chiang (1968) et détaillée par Preston et al. (200I).

Les probabilités de survie à la maladie $i,{ }_{n} p_{x}^{i}$ et celles pour toutes causes confondues nous ont permis de générer les tables de mortalité en l'absence de chaque maladie $i$ et pour chacune des périodes qui sont retenues. Les espérances de vie ainsi obtenues sont comparées selon les périodes et selon les causes $i$ de décès.

\section{Résultats}

\section{Niveaux de la mortalité}

Sur l'ensemble de la période, 224I hommes et 2186 femmes sont décédés pour 123750 personnesannées observées. De façon générale, pour les femmes, le niveau de mortalité a baissé dans tous les groupes d'âges entre les périodes 1997-2000 et 2009-2012 (Figure I), après avoir légèrement augmenté entre 1993-1996 et 1997-2000. L'espérance de vie à 50 ans augmente en moyenne de I,40 ans tous les quatre ans chez les femmes. La baisse de la mortalité n'est par contre pas observée chez les hommes, où l'évolution des taux a été erratique dans tous les groupes d'âges (Figure I). Entre le début et la fin de période, on constate même une augmentation entre 70-79 ans et les plus de 80 ans. L'espérance de vie à 50 ans a à peine évolué chez les hommes; elle augmente en moyenne de 0, 14 ans tous les 4 ans.

\section{Évolution de la répartition des causes de décès}

A partir des décès codifiés selon la CIMIO (OMS, 1993-1996) nous avons classé les causes de décès en quatre grands groupes : (I) maladies transmissibles; (2) maladies non transmissibles, (3) accidents et mortalité violente, (4) causes indéterminées. Les affections relatives à la grossesse sont généralement associées aux maladies transmissibles mais nous optons pour l'appellation unique de maladies transmissibles car nous n'avons dénombré que 5 cas de décès pour causes maternelles et les observations sur ces personnes ont été omises de la base des données. Les maladies transmissibles causant le plus de décès sont notamment le paludisme, les maladies respiratoires (pneumonie, toux/bronchite, laryngite), les maladies diarrhéiques, la tuberculose, la méningite et le $\mathrm{VIH} / \mathrm{SIDA}$. Les causes non transmissibles comprennent principalement les maladies cardiovasculaires (cardiopathie, accident vasculaire cérébral, hypertension artérielle), les maladies digestives, les tumeurs et le diabète. Le groupe des indéterminés comprend les causes mal définies. 


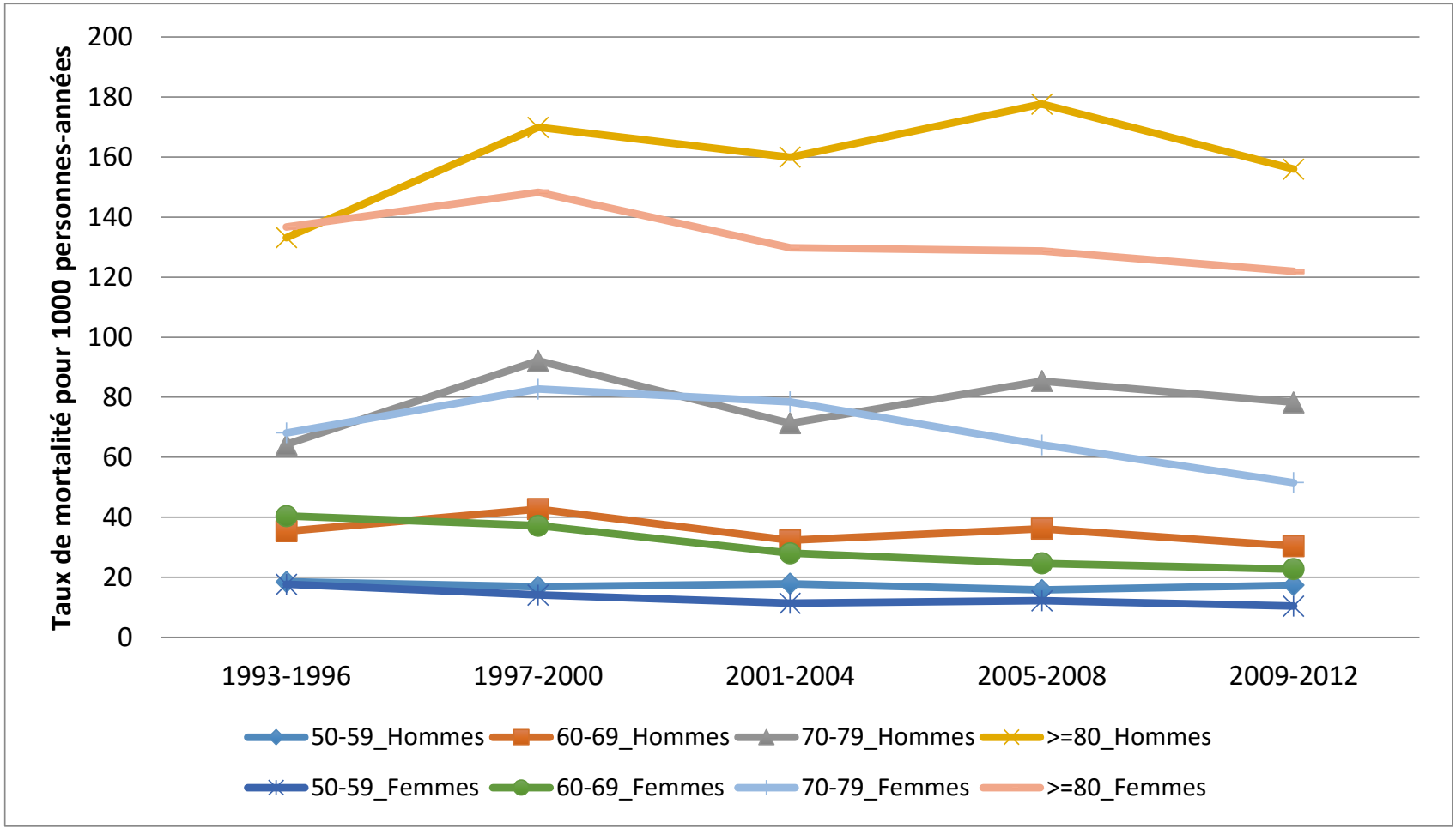

Figure I : Évolution des taux de mortalité (pour mille) par groupe d’âges, par période et par sexe entre 1993 et 2012

Tableau I : Evolution des proportions (\%) des causes spécifiques de décès au-delà de 50 ans pour la période de 1993-2012

\begin{tabular}{|c|c|c|c|c|c|c|}
\hline Causes de décès & $\begin{array}{l}1993-1996 \\
(n=116)(\%)\end{array}$ & $\begin{array}{l}1997-2000 \\
(n=433)(\%)\end{array}$ & $\begin{array}{l}200 I-2004 \\
(n=722)(\%)\end{array}$ & $\begin{array}{l}2005-2008 \\
(n=830)(\%)\end{array}$ & $\begin{array}{l}2009-2012(n=839) \\
(\%)\end{array}$ & $\begin{array}{l}\text { Ensemble } \\
(\mathrm{n}=2940) \\
(\%)\end{array}$ \\
\hline $\begin{array}{l}\text { Maladies } \\
\text { transmissibles }\end{array}$ & 56,0 & 61,7 & 55,1 & 52,9 & 48,9 & 53,6 \\
\hline Maladies diarrhéiques & 19,8 & 14,6 & 12,6 & 14,7 & 10,4 & 13,1 \\
\hline Méningite & $\mathrm{I}, 7$ & 0,9 & 0,4 & 2,1 & 3,1 & 1,8 \\
\hline Paludisme & 16,4 & 23,3 & 17,4 & 18,0 & 18,5 & 18,7 \\
\hline Maladies respiratoires & 18,1 & 17,3 & 19,5 & 14,8 & 11,8 & 15,6 \\
\hline $\mathrm{VIH} / \mathrm{SIDA}$ & 0,0 & 3,0 & 2,0 & 0,8 & 1,6 & 1,6 \\
\hline Tuberculose & 0,0 & 2,3 & 2,6 & 2,1 & 3,3 & 2,5 \\
\hline $\begin{array}{l}\text { Autres maladies } \\
\text { transmissibles }\end{array}$ & 0,0 & 0,3 & 0,6 & 0,4 & 0,2 & 0,3 \\
\hline $\begin{array}{ll}\text { Maladies } & \text { non } \\
\text { transmissibles } & \end{array}$ & 10,3 & 15,5 & 22,7 & 21,1 & 28,7 & 22,5 \\
\hline Tumeur/Cancers & 0,0 & 0,9 & 3,1 & 3,6 & 2,6 & 2,7 \\
\hline Diabète & 0,0 & 0,0 & 0,4 & 0,7 & 0,7 & 0,5 \\
\hline Épilepsie & 1,7 & 0,0 & 0,1 & 0,1 & 0,1 & 0,2 \\
\hline $\begin{array}{l}\text { Maladies } \\
\text { cardiovasculaires }\end{array}$ & 4,3 & 9,0 & 14,5 & 13,6 & 18,4 & 14,1 \\
\hline Maladies respiratoires & 0,0 & 0,2 & 0,7 & 0,7 & 0,2 & 0,5 \\
\hline Maladies digestives & 4,3 & 3,3 & 2,9 & 1,8 & 6,0 & 3,6 \\
\hline $\begin{array}{l}\text { Autres maladies non } \\
\text { transmissibles }\end{array}$ & 0,0 & 2,1 & 1,0 & 0,6 & 0,7 & 0,9 \\
\hline $\begin{array}{l}\text { Accidents et mortalité } \\
\text { violente }\end{array}$ & 3,5 & 4,6 & 2,9 & 3,0 & 1,4 & 2,8 \\
\hline Indéterminées & 30,2 & 18,2 & 19,3 & 23,0 & 21,0 & 21,1 \\
\hline Total & 100,0 & 100,0 & 100,0 & 100,0 & 100,0 & 100,0 \\
\hline
\end{tabular}


Le Tableau I présente la proportion des décès par grands groupes de causes utilisés dans cette étude au-delà de 50 ans. Entre les périodes 1993-1996 et 2009-2012, on observe dans l'ensemble une diminution de la part des maladies transmissibles qui est passée de $56,0 \%$ à $48,9 \%$ soit une baisse de $13,0 \%$ ( $p$-value $=0,158)$. Cette baisse s'est faite au profit des maladies non transmissibles, qui ont augmenté de manière très significative, de $10,3 \%$ à $28,7 \%$, soit une augmentation de $178,0 \%$ ( $p$ value $<0,00 \mathrm{I})$. La proportion de décès accidentels est passée de $3,5 \%$ à I, $4 \%$, soit une baisse de $58,0 \%$ ( $p$ value $=0,068$ ). Enfin, pour les indéterminés qui ont évolué de façon erratique, on est passé d'une proportion de $30,2 \%$ à $21,0 \%$, soit une baisse de $30,0 \%$ ( $p$-value $=0,029)$.

Sur l'ensemble de la période 1993-2012, les causes de décès les plus fréquentes sont respectivement le paludisme (18,7\%); les maladies respiratoires $(15,6 \%)$, les maladies cardiovasculaires $(14,1 \%)$ et les maladies diarrhéiques $(13,1 \%)$. Les tumeurs viennent en sixième position $(2,7 \%)$, après les maladies digestives chroniques $(3,6 \%)$. Les maladies spécifiques les plus fréquentes au sein des maladies cardiovasculaires sont la cardiopathie (59,6\%), les accidents vasculaires cérébraux $(23,3 \%)$ et l'hypertension artérielle (12,7\%). Dans le groupe des maladies respiratoires, les causes spécifiques sont notamment la pneumonie (88,4\%), la toux/bronchite $(10,5 \%)$ et la laryngite $(I, 1 \%)$. Pour les tumeurs, les pathologies spécifiques les plus fréquentes sont la tumeur maligne des organes génitaux $(29,5 \%)$, le cancer digestif $(21,8 \%)$ et la tumeur maligne du sein (II,5\%).

Toujours dans le Tableau I, il ressort que les maladies cardiovasculaires connaissent une augmentation significative notamment entre 1997$2000(9,0 \%)$ et $2009 / 2012(18,4 \%)$, soit une augmentation de $104,0 \%$ (p-value<0,00I). Par contre, la proportion des maladies respiratoires a diminué dans la même période, passant de $17,3 \%$ à ||$, 8 \%$, soit une baisse de $31,2 \%$ ( $p$-value $=0,0 \mid 4)$. Les proportions du paludisme oscillent sur l'ensemble de la période observée avec $16,4 \%$ en début de période en 1993-1997, $23,3 \%$ en 1997/2000 puis $18,5 \%$ en $2009 / 2012$. L'évolution des tumeurs permet de mettre en question la qualité des diagnostics par autopsies verbales, en particulier dans la première période, car on est passé de $0,0 \%$ en
$1993 / \mid 997$ puis $3,6 \%$ en $2005-2008$ et $2,6 \%$ en 2009/2012.

En examinant les proportions selon le sexe (résultats non présentés ici), il ressort que la contribution des maladies cardiovasculaires et respiratoires à la mortalité générale est plus élevée chez les hommes. La part des tumeurs dans la mortalité masculine progresse par ailleurs de façon quasiment linéaire sur toute la période d'étude. Par contre la contribution du paludisme à la mortalité générale est plus élevée chez les femmes.

\section{Évolution des taux de mortalité et contribution des causes spécifiques aux variations de l'espérance de vie à 50 ans}

Les Figures 2 et 3 présentent l'évolution des taux de mortalité par sexe pour différentes catégories de causes. Elles montrent que les taux de mortalité pour maladies cardiovasculaires sont plus élevés à toutes les périodes chez les hommes. Elles montrent aussi l'augmentation des taux de mortalité pour ces maladies, quel que soit le sexe. De 3,10 \%o en 19972000 chez les hommes, ce niveau a doublé pour atteindre 6,21\%० en 2009-2012. Chez les femmes, ces taux sont passés de $1,88 \%$ à $4,54 \%$ o sur la même période.

Pour les maladies respiratoires, on assiste à une augmentation des taux pour les deux sexes jusqu'à la période $200 \mathrm{I}-2004$ et ensuite à une baisse jusqu'à la dernière période (2009-20I2). Les taux de mortalité dus à cette pathologie sont également dans l'ensemble supérieurs chez les hommes. L'évolution de la mortalité palustre est quant à elle, très erratique chez les hommes. Chez les femmes, les taux de mortalité palustres enregistrent une baisse continue depuis la période 1997-2000.

Une autre façon de quantifier le poids des causes spécifiques de mortalité sur la mortalité dans une population est l'analyse de leur effet sur l'espérance de vie par la méthode de Chiang (1968). Cette approche permet de mettre en évidence une perte de 2,65 ans d'espérance de vie à 50 ans due à la montée des maladies cardiovasculaires entre 19972004 et 2005-2012. Par contre, pour les maladies respiratoires et le paludisme, la baisse des taux associés à ces pathologies correspond à des gains respectifs de 2,16 et 2,06 ans d'espérance de vie à 50 ans (Tableau 2). 


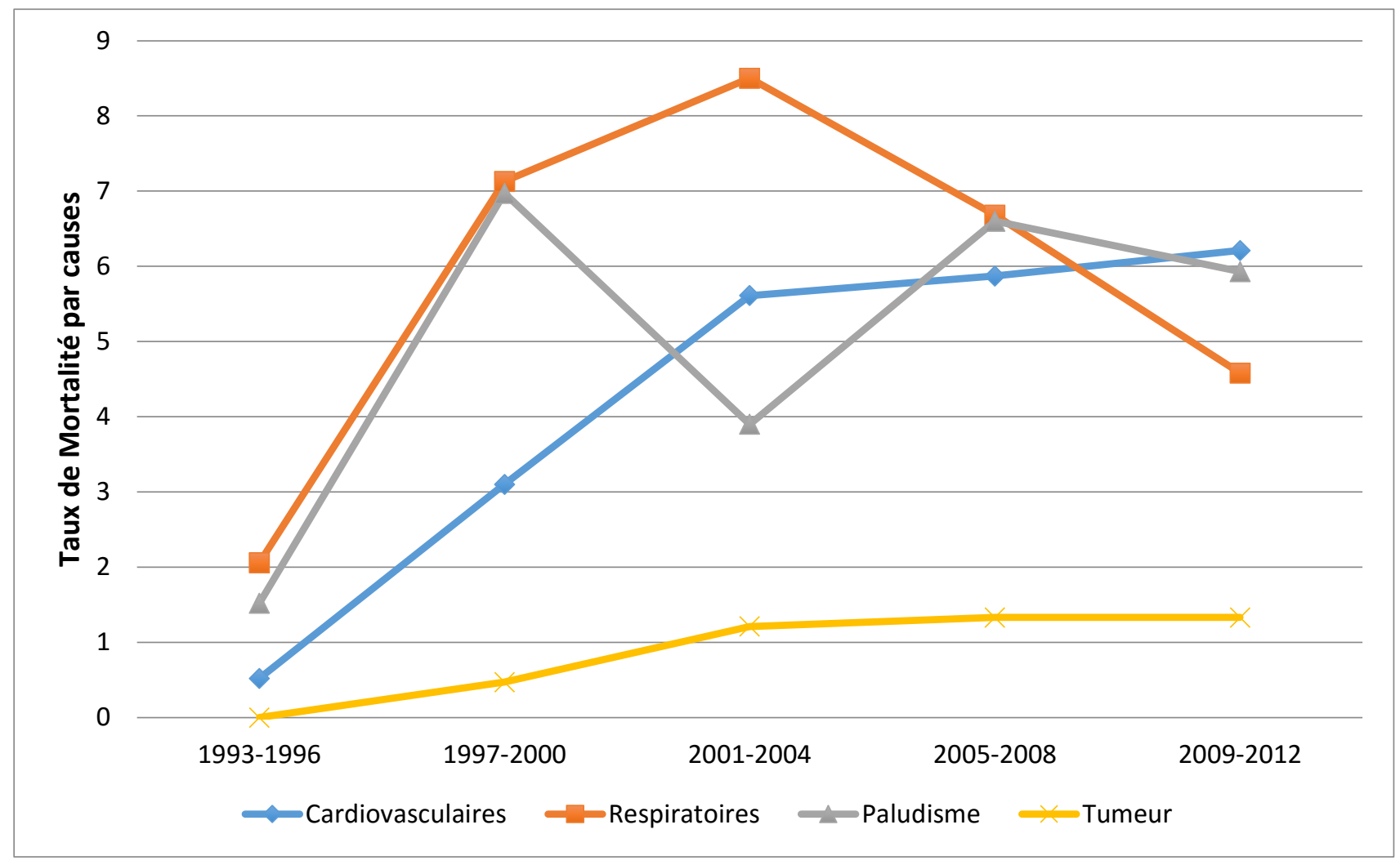

Figure 2 : Évolution des taux de mortalité des causes spécifiques de décès par période chez les hommes

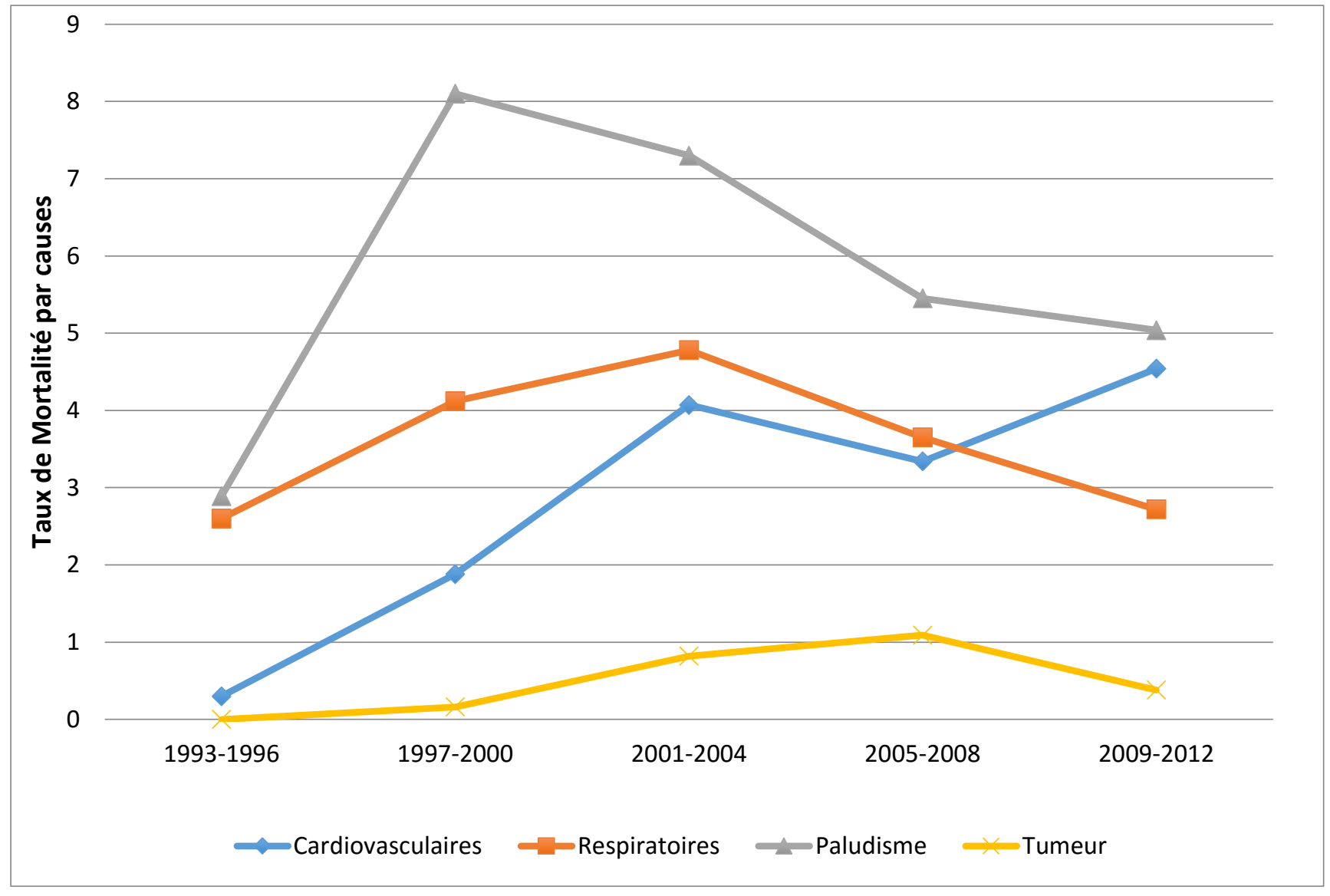

Figure 3 : Évolution des taux de mortalité des causes spécifiques de décès par période chez les femmes 
Tableau 2: Contribution des trois premières causes de décès aux variations de l'espérance de vie à 50 ans entre les périodes 1997-2004 et 2005-20I 2

\begin{tabular}{|c|c|c|c|c|}
\hline Groupes d'âges & $\begin{array}{l}\text { Maladies } \\
\text { cardiovasculaires }\end{array}$ & $\begin{array}{l}\text { Maladies } \\
\text { respiratoires }\end{array}$ & Paludisme & Total toutes causes \\
\hline 50-59 & $-0,72$ & $+0,70$ & $+0,35$ & 2,56 \\
\hline $60-69$ & $-0,72$ & $+0,70$ & $+0,50$ & 2,53 \\
\hline $70-79$ & $-0,56$ & $+0,56$ & $+0,48$ & 1,78 \\
\hline $80+$ & $-0,65$ & $+0,20$ & $+0,73$ & 0,81 \\
\hline Total tous âges & $-2,65$ & 2,16 & 2,06 & 7,68 \\
\hline
\end{tabular}

\section{Discussion}

Cette étude met en évidence des tendances en matière de niveaux de mortalité et de répartition des causes de décès qui vont dans le sens attendu. En effet, de nombreuses études ont déjà documenté la baisse de la mortalité en Afrique sub-saharienne chez les enfants et les jeunes adultes, même si l'évolution est plutôt erratique en ce qui concerne la mortalité aux âges avancés (Sie et al., 20I0; Kuate Defo, 20l4; Masquelier et al., 2014). Pour l'étude des causes de décès, il est également admis que les maladies infectieuses laissent de plus en plus de la place aux maladies non transmissibles, notamment chez les personnes âgées (Byass et al., 2010). Ce changement dans la hiérarchie des causes de décès résulte du vieillissement des populations, des succès dans la lutte contre les maladies infectieuses et parasitaires, mais il est également porté par des changements comportementaux qui favorisent le développement de maladies chroniques (sédentarité, tabagisme, alimentation trop riche en sucres et en graisses, etc.).

En ce qui concerne les causes spécifiques de décès, les résultats auxquels nous parvenons sont cohérents avec ceux publiés par Byass et al (2010) et Kynast-Wolf et al $(20 / 0)$ sur les causes de décès à Agincourt et à Nouna. En effet, la part de la mortalité due aux maladies cardiovasculaires augmente au fil du temps contrairement aux maladies respiratoires. A Nouna, les taux de mortalité dus aux maladies respiratoires ont commencé à diminuer au fil des années après un pic au cours de la période 2001 2004. La principale cause spécifique en jeu dans ce pic est la pneumonie $(88,0 \%)$. Les facteurs de risque importants de la pneumonie sont entre autres la consommation de tabac, d'alcool, ainsi que la prévalence des rhumes ou des grippes qui se prolongent à cause d'une basse température (Butler et Schuchat, 1999). Cette baisse de la mortalité due aux maladies respiratoires contrarierait, en tout cas pour les 50 ans et plus, les prévisions de Aboderin (20II), selon lesquelles les prochaines années verraient cette pathologie connaître une augmentation. En matière de paludisme, rappelons que les études réalisées sur les causes de décès au
Burkina Faso sont unanimes pour indiquer que le paludisme demeure la première cause de mortalité générale et ce, y compris aux âges avancés (Ramroth et al., 20I2; Soura et al., 2014). La mortalité due aux tumeurs dans notre étude, n'est que la troisième cause de décès parmi les maladies non transmissibles bien qu'elle augmente de façon générale en termes de proportions et de taux. Une des explications possibles de la rareté des décès par tumeurs dans notre population pourrait être le diagnostic difficile de cette pathologie par les autopsies verbales, en particulier dans la première période d'observation. Certains décès dus à cette cause à Nouna ont probablement été mal classés dans d'autres catégories de maladies non transmissibles ou parmi les causes inconnues (Streatfield et al., 20l4b).

En confinant l'analyse aux plus de 50 ans, nous montrons que l'importance croissante des causes non transmissibles ne résulte pas uniquement de l'augmentation du nombre de personnes âgées mais est également associé à des facteurs comportementaux des individus. Sous l'influence de la modernisation, les changements dans les facteurs de risque associés aux comportements des individus sont nombreux (Bygbjerg, 20I2) : consommation de tabac, d'alcool, mauvaise alimentation, la sédentarité. De manière indirecte, ces différents facteurs de risque influent notamment sur l'hypertension artérielle. Dans un contexte de faible accès aux soins de santé, ces facteurs pourraient entraîner une montée de mortalité précoce par maladie cardiovasculaire. Les facteurs comportementaux permettent aussi d'expliquer une grande part des différences entre les hommes et les femmes adultes: en particulier, la consommation d'alcool, un facteur de risque majeur dans les maladies du foie, est fréquent chez les hommes adultes (Duthé et Pison, 2008). Ces maladies non transmissibles sont également davantage liées à la diminution de la capacité des individus à préserver l'équilibre de fonctionnement de leur organisme, conséquence qui accompagne le vieillissement du corps. Elles reflèteraient ainsi le processus de sénescence (Horiuchi, 2007), c'est-à-dire que la lente dégradation des fonctions physiologiques des individus qui 
accompagne le vieillissement et, de surcroit, rend les individus davantage vulnérables à certaines pathologies.

La principale question qui ressort après une analyse des données des autopsies verbales est évidemment la validité de ces résultats, étant donné les limitations des données en quantité (faiblesse des effectifs) et en qualité (fiabilité relative des autopsies verbales) (Garenne. et al., 1999). La classification des causes de décès n'est pas simple, surtout aux âges élevés, parce que la mortalité est généralement le résultat d'un processus complexe et la méthode des autopsies verbales ne peut pas remplacer une autopsie médicale. Les biais qui entachent les autopsies verbales peuvent se produire à différents stades: le questionnaire utilisé, l'entretien, le diagnostic ou la classification des décès (Soleman et al., 2006).

Ces problèmes se posent avec davantage d'acuité pour les décès au-delà de 50 ans. Les causes de décès des adultes et des personnes âgées sont multiples, et elles peuvent être associées. En ce sens, elles sont généralement plus difficiles à déterminer que celles des enfants (Chandramohan et al., 1994). De manière générale, les études confirment que les maladies infectieuses, les causes maternelles, ainsi que les accidents sont mieux décelés que les maladies non transmissibles. Toutefois, la plupart des études s'accordent sur l'intérêt de la méthode pour mesurer le poids de grands groupes de maladies (Chandramohan et al., 1998). En effet, les erreurs de classification interviennent généralement au sein des mêmes groupes (cardiovasculaire, respiratoire, digestif...) (Yang et al., 2006). Or, les facteurs de risque étant souvent les mêmes au sein de ces groupes, ces recherches permettent tout de même d'identifier les priorités en matière de santé publique (Garenne. et al., 1999).

Au-delà des problèmes de classification des décès via la méthode des autopsies verbales, la principale limite de l'analyse menée ici est qu'elle ne fait en fait référence qu'à $52,5 \%$ des décès, car les autres décès n'ont pas pu être suivis dans les temps par un entretien auprès des proches. $71,0 \%$ des décès qui n'ont pas de cause clairement définie correspondent à des cas où une autopsie verbale n'a pas été faite. Nous avons fait ici l'hypothèse selon laquelle il y a indépendance entre la conduite de l'autopsie verbale et la cause de décès sous-jacente. Cette hypothèse est valable dans la mesure où nous pensons que des processus aléatoires sont à l'origine de la nonconduite des autopsies verbales. Les causes qui pouvaient leur être attribuées ne devraient donc pas changer fondamentalement les tendances de nos indicateurs. II faut toutefois relever les limites des suivis de population qui résident dans le fait que l'observation est limitée par une fenêtre spatiotemporelle; les informations disponibles sur certains individus peuvent être incomplètes parce que ces personnes ont émigré ou ont disparu de notre population sous surveillance (Courgeau et Lelièvre, 1989). Ces attritions peuvent avoir des effets sur nos résultats au cas où elles sont élevées ou si les personnes qui sortent sont sélectionnées. Toutefois, le niveau de l'attrition n'est pas très élevé à Nouna et ne devrait pas influencer de façon significative nos indicateurs. Le taux d'attrition varierait selon l'année de $3,6 \%$ à $9,1 \%$ (Sie et al., 2010), tous âges confondus.

En revanche, les $29,0 \%$ restants qui sont des cas où des autopsies verbales ont été réalisées mais il n'y a pas eu de consensus entre les médecins pour une cause définitive de décès pourraient affecter les tendances que nous avons rapportées. L'effet de ces causes classifiées comme indéterminées sur nos tendances pourrait néanmoins être moindre par le fait que pour les 4 périodes entre 1997 et 20I2, leurs pourcentages sont restés relativement constants, se situant entre $18 \%$ et $23 \%$. La part importante des décès dont les causes sont mal définies est due au fait que nous nous intéressons qu'aux personnes âgées. C'est en effet aux âges avancés que la part des décès de cause mal définie ( $y$ compris la sénilité) est la plus importante (Waltisperger et Meslé, 2005). La proportion de décès de cause mal définie varie généralement de $20 \%$ jusqu'à $50 \%$ au-delà de 60,70 ou 75 ans selon les études (Joshi et al., 2006, Kahn et al., 1999, Duthe et al., 2009, Byass et al., 2010, Soura et al., 20l4, Herbst et al., 20II, Ramroth et al., 20I2). En présence de statistiques incomplètes, il est d'usage de tenir compte des causes mal définies en redistribuant ces décès dans les autres catégories de causes (Waltisperger et Meslé, 2005) mais vu leur proportion élevée, chercher un moyen de redistribuer ces causes sans faire une hypothèse simple de proportionnalité comme nous l'avons fait semble vain (Duthe et al., 2009). Certaines causes de décès étant plus faciles que d'autres à diagnostiquer, la distribution des causes réelles de décès peut être différente de celle des causes connues. En exemple, les tumeurs, certaines maladies infectieuses autres que la tuberculose ou encore certaines maladies rares, ou liées à la dégénérescence, constituent une catégorie de cause difficile à identifier, contrairement aux accidents. On peut donc s'attendre à une surestimation du poids des maladies faciles à identifier par rapport aux autres (Duthe et al., 2009) et les taux de mortalité par cause sont à prendre avec précaution. II n'en reste pas moins que l'examen des taux de mortalité par cause est nécessaire (Kahn et al., 1999; Streatfield, P. K et al., 20I4; Soura, B. et al., 2014). 
Enfin, insistons sur le caractère local de l'information recueillie par les observatoires. Si le contexte de la zone d'étude de Nouna ressemble à maints autres lieux dans les pays sahéliens, la représentativité des données au sens statistique ne va pas au-delà des frontières de la zone étudiée; ainsi il faut être prudent lorsqu'on généralise les résultats au niveau régional ou national (Tabutin et Casseli, 2006; Chandramohan et al., 2008). Pour mieux estimer les paramètres nationaux, des sources de données complémentaires sont donc nécessaires (Hammer et al., 2006). Dans certains pays, où plusieurs observatoires sont mis en place comme le Sénégal, le Burkina Faso ou la Tanzanie, l'emplacement des observatoires couvre différentes régions ou conditions socio-économiques. En constituant un réseau d'observatoires, on peut alors remédier en partie à la non-représentativité de chacun en ayant une idée de la gamme de variations à l'intérieur du pays (Tabutin et Casseli, 2006). Ce type de travail collaboratif est encore à ses débuts au Burkina Faso.

Malgré ces différentes limites, les systèmes de surveillance démographique et de santé demeurent jusqu'à présent les seules méthodes viables pour estimer les causes de décès dans de nombreuses régions d'Afrique et d'Asie.

\section{Conclusion}

La population de Nouna, zone rurale du Burkina Faso, a subi des changements rapides en termes de morbidité et de mortalité des personnes âgées. Les personnes âgées de 50 ans et plus dans cette zone souffrent des maladies transmissibles (paludisme et maladies respiratoires) et non transmissibles (maladies cardiovasculaires et tumeurs). La première catégorie de causes de décès dans cette population demeure les maladies transmissibles avec néanmoins une montée rapide des maladies non transmissibles. Ce double fardeau de maladies au sein de cette population appelle des actions spécifiques de la part des pouvoirs publics.

\section{Remerciements}

Les auteurs tiennent à remercier le Programme interuniversitaire de formation en recherche en Santé Mondiale (Santé-Cap) pour leur soutien financier reçu par L. Niamba durant les deux premières années de sa formation doctorale. Nos remerciements vont également à toute la communauté du Système de Surveillance Démographique et de Santé de Nouna, aux leaders communautaires, informateurs clés, enquêteurs, superviseurs et agents de saisie du Centre de Recherche en Santé de Nouna.

\section{Bibliographie}

Aboderin, I. (20I0). Understanding and advancing the health of older populations in sub-Saharan Africa: policy perspectives and evidence needs. Pub Health Rev, 32(2), 357-376.

Baya, B. (2004). Population and infectious diseases in Burkina Faso. In: IUSSP (ed.) Second seminar of the IUSSP Scientific Committee on emerging health threats HIV, resurgent infections, and population change in Africa. Ouagadougou, Burkina Faso.

Butler, J. C. \& Schuchat, A. (1999). Epidemiology of pneumococcal infections in the elderly. Drugs \& aging I5(I), II-19.

Byass, P., Kahn, K., Fottrell, E., Collinson, M. A. \& Tollman, S. M. (20/0). Moving from data on deaths to public health policy in Agincourt, South Africa: approaches to analysing and understanding verbal autopsy findings. PLoS Med, 7(8), el 000325.

Bygbjerg, I. C. (20/2). Double burden of noncommunicable and infectious diseases in developing countries. Science, 337(6101), 1499I50I.

Chandramohan, D., Maude, G. H., Rodrigues, L. C. \& Hayes, R. J. (1994). Verbal autopsies for adult deaths: issues in their development and validation. International Journal of Epidemiology , 23(2), 213222.

Chandramohan, D., Maude., G. H., Rodrigues, L. C. \& Hayes, R. J. (1998). Verbal autopsies for adult deaths: their development and validation in a multicentre study. Trop Med Int Health, 3(6), 436-446.

Chandramohan, D., Shibuya, K., Setel, P., Cairncross, S., Lopez, A. D., Murray, C. J., Zaba, B., Snow, R. W. \& Binka, F. (2008). Should data from demographic surveillance systems be made more widely available to researchers? PLoS Med, 5(2), e57.

Courgeau, D. \& Lelièvre, E. (1989). Analyse démographique des biographies, Ined.

Aikins, A. D. G., Unwin, N., Agyemang, C., Allotey, P., Campbell, C. \& Arhinful, D (20I0). Tackling Africa's chronic disease burden: from the local to the global. Globalization and Health, 6(I), I.

Defo, B. K. (20I4). Demographic, epidemiological, and health transitions: are they relevant to population health patterns in Africa? Glob Health Action, 7.

Duthé, G., Laurent, R. \& Pison, G. (2009). Vivre et mourir après 60 ans en milieu rural africain, Ined.

Duthé, G. \& Pison, G. (2008). Adult mortality in a rural area of Senegal: Non-communicable diseases have a large impact in Mlomp. Demographic Research, 19, 1419-1434. 
Duthé, G., Pison, G. \& Laurent, R. (20I0). Situation sanitaire et parcours de soins des personnes âgées en milieu rural africain: Une étude à partir des données du suivi de population de Mlomp (Sénégal). Autrepart, (I), I67-I87.

Garenne., M., Tollman., S., Kahn., K. \& Gear., J. (1999). Causes de décès dans une zone rurale d'Afrique du Sud comparées à deux autres situations (Sénégal et France), (No. 54). Ceped.

Hammer, G. P., Kouyate, B., Ramroth, H. \& Becher, H. (2006). Risk factors for childhood mortality in sub-Saharan Africa: a comparison of data from a Demographic and Health Survey and from a Demographic Surveillance System. Acta Trop, 98(3), 2। 2-2।8.

Herbst, A. J., Mafojane, T. \& Newell, M. L. (20I I). Verbal autopsy-based cause-specific mortality trends in rural KwaZulu-Natal, South Africa, 20002009. Popul Health Metr, 9(47), 0-4.

Horiuchi, S. (2007). Causes of death among the oldest-old: age-related changes in the causes-ofdeath distribution. In Human Longevity, individual life duration, and the growth of the oldest-old population (pp. 2 I5-235). Springer Netherlands.

Joshi, R., Cardona, M., lyengar, S., Sukumar, A., Raju, C. R., Raju, K. R., Raju, K., Reddy, K. S., Lopez, A. \& Neal, B. (2006). Chronic diseases now a leading cause of death in rural India-mortality data from the Andhra Pradesh Rural Health Initiative. Int J Epidemiol, 35(6), I522-I529.

Kahn, K., Tollman, S. M., Garenne, M. \& Gear, J. S. (1999). Who dies from what? Determining cause of death in South Africa's rural north-east. Tropical Medicine and International Health, 4(6), 433-44I.

Mackenbach, J. P. (1994). The epidemiologic transition theory. J Epidemiol Community Health, 48(4), 329-331.

Maher, D. \& Sekajugo, J. (20I I). Research on health transition in Africa: time for action. Health Res Policy Syst, 9(5), I0-I I 86.

Masquelier, B., Waltisperger, D., Ralijaona, O., Pison, G. \& Ravelo, A. (20/4). The epidemiological transition in Antananarivo, Madagascar: an assessment based on death registers (I900-20I2). Glob Health Action, 7.

Misganaw, A. (20/2). The double mortality burden among adults in Addis Ababa, Ethiopia, 20062009. Preventing Chronic Disease, 9.

Murray., C. \& Lopez., A. L. (20/2). Global and regional mortality from 235 causes of death for 20 age groups in 1990 and 2010: a systematic analysis for the Global Burden of Disease Study 2010. Lancet, 380(9859), 2095-2 28.

Olshansky., S. \& Ault., B. (1986). The fourth stage of the epidemiologic transition: the age of delayed degenerative diseases. The Milbank Quaterly, 355-391.

Omran, A. R. (197I). The epidemiological transition: a theory of the epidemiology of population change. The Milbank Memorial Fund Quarterly, 49(4), 509-538.

Organisation Mondiale de la Santé(OMS) (19931996). Classification internationale des maladies, IOème révision (1992-1994). OMS, Génève.

United Nations (20I4). World Population Prospects: The 2012 Revision, excel tables-population data [Online].

Preston, S. H., Heuveline, P. \& Guillot, M. (200I). Demography: measuring and modeling population processes. Pop. Dev. Rev, 27, 365.

Ramroth, H., Lorenz, E., Rankin, J. C., Fottrell, E., Yé, M., Neuhann, F., Ssennono, M., Sié, A., Byass, P. \& Becher, $H$. (20I2). Cause of death distribution with InterVA and physician coding in a rural area of Burkina Faso. Trop Med Int Health, I7(7), 904913.

Rossier, C., Soura, A. B., Duthé, G. \& Findley, S. (20/4). Non-Communicable disease mortality and risk factors in formal and informal neighborhoods, Ouagadougou, Burkina Faso: evidence from a Health and Demographic Surveillance System. PLoS One, 9(I2), el I 3780.

Rowe, A. K. (2006). Analysis of deaths with an unknown cause in epidemiologic analyses of mortality burden. Trop Med Int Health, I I(4), 540-550.

Salomon, J. A., Wang, H., Freeman, M. K., Vos, T., Flaxman, A. D., Lopez, A. D. \& Murray, C. J. L. (20/3). Healthy life expectancy for 187 countries, 1990-2010: a systematic analysis for the Global Burden Disease Study 2010. Lancet, 380(9859), 2144-2162.

Sié, A., Louis, V. R., Gbangou, A., Muller, O., Niamba, L., Stieglbauer, G., Ye, M., Kouyate, B., Sauerborn, R. \& Becher, H. (20I0). The Health and Demographic Surveillance System (HDSS) in Nouna, Burkina Faso, 1993-2007. Glob Health Action, 3.

Soleman, N., Chandramohan, D. \& Shibuya, K. (2006). Verbal autopsy: current practices and challenges. Bulletin of the WHO 84(3), 239-245.

Soura, A. B., Lankoande, B., Millogo, R. \& Bangha, M. (20/4). Comparing causes of death between formal and informal neighborhoods in urban Africa: evidence from Ouagadougou Health and Demographic Surveillance System. Glob Health Action, 7.

Tollman, S. M., Kahn, K., Sartorius, B., Collinson, M. A., Clark, S. J., \& Garenne, M. L. (2008). Implications of mortality transition for primary health care in rural South Africa: a population- 
based surveillance study. Lancet, 372(9642), 893901.

Streatfield, P. K., Khan, W. A., Bhuiya, A., Alam, N., Sié, A., Soura, A. B., Bonfoh, B., Ngoran, E. K., Weldearegawi, B., Jasseh, M., Oduro, A., Gyapong, M., Kant, S., Juvekar, S., Wilopo, S., Williams, T. N., Odhiambo, F. O., Beguy, D., Ezeh, A., Kyobutungi, C., Crampin, A., Delaunay, V., Tollman, S. M., Herbst, K., Chuc, N. T., Sankoh, O. A., Tanner, M. \& Byass, P. (20I4a). Cause-specific mortality in Africa and Asia: evidence from INDEPTH Health and Demographic Surveillance System sites. Glob Health Action, 7.

Streatfield, P. K., Khan, W. A., Bhuiya, A., Hanifi, S. M., Alam, N., Bagagnan, C. H., Sié, A., Zabre, P., Lankoande, B., Rossier, C., Soura, A. B., Bonfoh, B., Kone, S., Ngoran, E. K., Utzinger, J., Haile, F., Melaku, Y. A., Weldearegawi, B., Gomez, P., Jasseh, M., Ansah, P., Debpuur, C., Oduro, A., Wak, G., Adjei, A., Gyapong, M., Sarpong, D., Kant, S., Misra, P., Rai, S. K., Juvekar, S., Lele, P., Bauni, E., Mochamah, G., Ndila, C., Williams, T. N., Laserson, K. F., Nyaguara, A., Odhiambo, F. O., Phillips-Howard, P., Ezeh, A., Kyobutungi, C., Oti, S., Crampin, A., Nyirenda, M., Price, A., Delaunay, V., Diallo, A., Douillot, L., Sokhna, C.,
Gomez-Olive, F. X., Kahn, K., Tollman, S. M., Herbst, K., Mossong, J., Chuc, N. T., Bangha, M., Sankoh, O. A. \& Byass, P. (20|4b). Adult noncommunicable disease mortality in Africa and Asia: evidence from INDEPTH Health and Demographic Surveillance System sites. Glob Health Action, 7.

Tabutin, D. \& Casseli, G. (2006). Les systèmes de collecte des données en démographie.

Tabutin, D. \& Schoumaker, B. (2004). La démographie de l'Afrique au sud du Sahara, des années 1950 aux années 2000.. Population, 59(3), $521-622$.

Vallin, J. (2002). The End of the demographic transition: relief or concern? Population and Development Review, 28(I), I05-I 20.

Vallin, J. \& Meslé, F. (20/0). De la transition épidemiologique à la transition sanitaire: l'improbable convergence générale. Ralentissements, résistances, ruptures dans les transitions démographiques: Actes de la Chaire Quetelet 2010, 257.

Waltisperger, D. \& Meslé, F. (2005). Crise économique et mortalité. Population, 60(3), 243275.

Yang, G., Rao, C., Ma, J., Wang, L., Wan, X., Dubrovsky, G. \& Lopez, A. D. (2006). Validation of verbal autopsy procedures for adult deaths in China. Int J Epidemiol, 35(3), 74 I-748. 